\title{
Eradication therapy for Helicobacter pylori infection improves nutrition status in Japanese hemodialysis patients: a pilot study
}

\author{
Hitomi Ichikawa, ${ }^{1}$ Mitsushige Sugimoto, ${ }^{2, *}$ Yukitoshi Sakao, ${ }^{3}$ Shu Sahara, ${ }^{1}$ Naro Ohashi, ${ }^{1}$ Koji Sano, ${ }^{4}$ \\ Shigeru Tadokoro, ${ }^{5}$ Hisanori Azekura, ${ }^{6}$ Akira Shimomura, ${ }^{7}$ Fuyuki Yamashita, ${ }^{8}$ Daiki Sugiyama, ${ }^{9}$ Ken Fukuta, ${ }^{10}$ \\ Takahisa Furuta, ${ }^{11}$ Akihiko Kato, ${ }^{12}$ Ken Sugimoto $^{1}$ and Hideo Yasuda ${ }^{1}$ \\ ${ }^{1}$ First Department of Medicine, Hamamatsu University School of Medicine, 1-20-1 Handayama, Higashi-ku, Hamamatsu, Shizuoka 431-3192, Japan \\ ${ }^{2}$ Division of Digestive Endoscopy, Shiga University of Medical Science Hospital, Seta, Tsukinowa-cho, Otsu, Shiga 520-2192, Japan \\ ${ }^{3}$ Hamana Clinic, 235-1 Numa, Hamakita-ku, Hamamatsu, Shizuoka 434-0037, Japan \\ ${ }^{4}$ Sano Clinic, 1818 Tennou-cho, Higashi-ku, Hamamatsu, Shizuoka 435-0052, Japan \\ ${ }^{5}$ Tadokoro Clinic, 1239 Uchino, Hamakita-ku, Hamamatsu, Shizuoka 434-0044, Japan \\ ${ }^{6}$ Sanaru Sun Clinic, 2-14-39 Higashiiba, Naka-ku, Hamamatsu, Shizuoka 432-8036, Japan \\ ${ }^{7}$ Sanarudai Asahi Clinic, 5-20-10 Sanarudai, Naka-ku, Hamamatsu, Shizuoka 432-8021, Japan \\ ${ }^{8}$ Yamashita Clinic, 2-1-5 Nakaizumi, Iwata, Shizuoka 438-0078, Japan \\ ${ }^{9}$ Satsuki no Mori Clinic, 1665-2 Nakase, Hamakita-ku, Hamamatsu, Shizuoka 434-0012, Japan \\ ${ }^{10}$ Hiryu Clinic, 304-9 Akura, Futamata-cho, Tenryu-ku, Hamamatsu, Shizuoka 431-3311, Japan \\ ${ }^{11}$ Center for Clinical Research, Hamamatsu University School of Medicine, 1-20-1 Handayama, Higashi-ku, Hamamatsu, Shizuoka 431-3192, Japan \\ ${ }^{12}$ Blood Purification Unit, Hamamatsu University School of Medicine, 1-20-1 Handayama, Higashi-ku, Hamamatsu, Shizuoka 431-3192, Japan
}

(Received 25 May, 2018; Accepted 25 June, 2018; Published online 2 October, 2018)

Plasma ghrelin level is influenced by Helicobacter pylori ( $H$. pylori) status and the severity of gastric mucosal atrophy, and the ghrelin level is associated with nutrition status in hemodialysis patients. Here, we investigated the efficacy of $H$. pylori eradication therapy in improving nutrition status in relation to the ghrelin level in $H$. pylori-positive hemodialysis patients. Of $H$. pylori-positive patients receiving hemodialysis at $\mathbf{8}$ dialysis center, $\mathbf{2 1}$ patients underwent gastroduodenoscopy for evaluation of the severity of gastric atrophy, and nutrition markers and plasma ghrelin levels before and 1 year after $\boldsymbol{H}$. pylori eradication therapy were evaluated. Serum cholinesterase level was significantly increased after $\boldsymbol{H}$. pylori eradication compared with the level before eradication (303.2 \pm 76.0 vs $287.3 \pm 68.1 \mathrm{IU} / \mathrm{L}, p=0.029)$. In particular, cholesterol (before, $196.6 \pm 23.2 \mathrm{mg} / \mathrm{dl}$; after, $206.1 \pm 25.9 \mathrm{mg} / \mathrm{dl}, p=$ 0.042 ) and cholinesterase levels (before, $296.9 \pm 70.8 \mathrm{IU} / \mathrm{L}$; after, $316.4 \pm 73.8 \mathrm{IU} / \mathrm{L}, p=0.049$ ) increased more strongly in patients with mild-moderate atrophy than those with severe atrophy, irrespective of improvement of plasma acyl-ghrelin and desacylghrelin levels after eradication therapy. In conclusion, $\boldsymbol{H}$. pylori eradication may improve nutrition status by increasing serum cholinesterase and cholesterol levels in hemodialysis patients, especially those with mild and moderate gastric mucosal atrophy.

Key Words: ghrelin, nutritional marker, eradication, Helicobacter pylori, hemodialysis

$\boldsymbol{T}$ he state of metabolic and nutritional derangement, called protein-energy wasting (PEW), has a major impact on mortality in hemodialysis patients. ${ }^{(1,2)}$ In general, PEW causes severe and complex processes of muscle loss, poor food intake, inflammation, and cardiovascular disease pathways. ${ }^{(3)}$ Improving the prognosis of hemodialysis patients with $\mathrm{PEW}$ therefore requires clarification of the biological mechanism of PEW development and the establishment of countermeasures against the development of PEW.

Ghrelin, an orexigenic peptide produced mainly by X/A-like cells in the stomach, is a major factor associated with malnutrition in general patients and with the pathogenesis of PEW in hemo- dialysis patients. ${ }^{(3-5)}$ Ghrelin has multiple favorable functions, including enhancement of orexigenic effect, protein anabolism, anti-inflammatory actions, a sensor of systemic oxidative stress, increase of preprandial acid secretion and cardiovascular protection. ${ }^{(5-9)}$ Recently, in addition, ghrelin is shown to stimulate food intake and to play gastric motility via expression of gastric ghrelin-induced c-kit protein in patients with functional dyspepsia and animal model. ${ }^{(10-13)}$ Hemodialysis patients with a low ghrelin level are at increased risk of cardiovascular mortality, ${ }^{(14)}$ and recent clinical trials show that administration of ghrelin improves malnutrition by increasing appetite and food intake. ${ }^{(15,16)}$ Therefore, both nutrition status and prognosis in hemodialysis patients are correlated with ghrelin levels.

Helicobacter pylori (H. pylori) infection affects the incident rate of gastroduodenal disease and nutritional status. ${ }^{(17,18)} \mathrm{H}$. pylori eradication therapy often causes individuals with nomal renal function to develop hyperlipidemia and hyperproteinaemia, along with an increase of body weight and body mass index (BMI). ${ }^{(19)}$ This phenomenon is considered to be due to increases in plasma ghrelin level followed by increased appetite and food intake after H. pylori eradication therapy. ${ }^{(20,21)}$ In general, ghrelin is downregulated by $H$. pylori infection and up-regulated by $H$. pylori eradication therapy. Biological roles of gastric and plasma ghrelin are altered in response to $H$. pylori infection and according to time-course expression levels of ghrelin are decreasing in relation with severity of gastric mucosal atrophy. ${ }^{(22)}$ Previously, we reported that plasma ghrelin level was significantly lower in hemodialysis patients with $H$. pylori infection than in those without this condition, and was inversely correlated with the severity of gastric mucosal atrophy in $H$. pylori-positive patients. ${ }^{(23)}$ We hypothesized that ghrelin level increases after eradication therapy and that nutritional status and prognosis are improved in hemodialysis patients after $H$. pylori eradication.

In this study, we clarified infection rates of $H$. pylori in Shizuoka, Japan, and investigated plasma ghrelin levels and

*To whom correspondence should be addressed. E-mail: sugimo@belle.shiga-med.ac.jp 
nutritional markers before and after $H$. pylori eradication therapy in $H$. pylori-positive Japanese hemodialysis patients.

\section{Materials and Methods}

Approval for the study protocol was provided by the Institutional Review Board of Hamamatsu University School of Medicine (Number: 23-185) and all patients provided written informed consent before enrollment. The registration number of this clinical research was University hospital Medical Information Network (UMIN) 000023336.

Subjects. The study enrolled patients receiving maintenance hemodialysis at 8 dialysis centers in Shizuoka, Japan (Sano Clinic, Tadokoro Clinic, Sanaru Sun Clinic, Sanarudai Asahi Clinic, Yamashita Clinic, Satsuki no Mori Clinic, Hiryu Clinic and Hamana Clinic). All patients had received regular hemodialysis for $4 \mathrm{~h}$ three times per week for at least 1 month. Exclusion criteria were a history of $H$. pylori eradication, history of gastrectomy, significant clinical illness (e.g., cancer), lack of informed consent and non-dialysis.

Hemodialysis patients who were serologically positive for $H$. pylori (anti-H. pylori antibody titer of more than 10) and provided informed consent for $H$. pylori eradication therapy underwent gastroduodenoscopy before eradication therapy. Blood samples were taken for measurement of plasma acyl- and desacyl-ghrelin levels, nutrition markers (serum albumin, total cholesterol and cholinesterase) and an inflammation marker [serum C-reactive protein (CRP)].

Eradication of $H$. pylori was performed by triple therapy with esomeprazole $20 \mathrm{mg}$, amoxicillin $250 \mathrm{mg}$ or $750 \mathrm{mg}$, and clarithromycin $200 \mathrm{mg}$ twice daily for 1 week. After therapy, success of eradication was confirmed by the ${ }^{13} \mathrm{C}$-urea breath test conducted at least 4 weeks after the end of eradication therapy.

One year after confirmed eradication, patients underwent gastroduodenoscopy and blood samples were taken for measurement of ghrelin, nutrition markers and CRP. As an index of nutritional status, we calculated the geriatric nutritional risk index (GNRI). ${ }^{(24,25)}$ If the dry weight after hemodialysis is higher than the ideal body weight, we calculated GNRI with dry weight/ideal body weight as $1 .{ }^{(24,25)}$

Assay of plasma ghrelin levels. Blood samples were taken from all fasted patients in the morning. Samples were collected in an aprotinin/EDTA-containing tube and immediately centrifuged at $1,500 \times g$ for $15 \mathrm{~min}$ at $4^{\circ} \mathrm{C}$. The resulting plasma was agitated with the addition of $1 \mathrm{~mol} / \mathrm{L}$ hydrochloric acid equal to one-tenth of the plasma volume and stored at $-80^{\circ} \mathrm{C}$ until assay. We used a two-site sandwich enzyme-linked immunosorbent assay specific for acyl-ghrelin and desacyl-ghrelin (Active Ghrelin ELISA Kit and Des-acyl Ghrelin ELISA Kit; SCETI, Tokyo, Japan).

Diagnosis of $\boldsymbol{H}$. pylori infection and assessment of gastric mucosal atrophy. H. pylori infection was diagnosed using an anti-H. pylori IgG serological test (E plate Eiken $H$. pylori antibody; Eiken Chemical Co. Ltd., Tochigi, Japan). Patients were considered positive when the anti- $H$. pylori antibody titer was more than 10. The endoscopic gastric mucosal atrophic pattern was evaluated according to the Kimura-Takemoto classification. ${ }^{(26)}$ Severity of atrophy was categorized into the four groups of non-atrophic; mild, Close (C)-I and C-II; moderate, C-III and Open (O)-I; and severe, O-II and O-III.

Assay of serum pepsinogen levels. Serum levels of pepsinogen (PG) I and PG II were measured using a commercially available kit (Pepsinogen CLEIA; Fuji Rebio Ltd., Tokyo, Japan) by chemiluminescence enzyme immunoassay (EIA), and PG I/II ratio was calculated as a serological marker of gastric mucosal atrophy. ${ }^{(27)}$

Statistical analyses. Normally numerical values were expressed as mean \pm SD. The comparison of clinical parameters before and after $H$. pylori eradication therapy was assessed using paired $t$ test or one-way ANOVA. Categorical variables were compared using the Chi-square test. All $p$ values were two-sided. $P<0.05$ was considered statistically significant. Calculations were carried out using StatView 5.0 statistical software (SAS Institute, Cary, NC).

\section{Results}

Patient characteristics. Of Japanese hemodialysis patients in this multicenter study, $14.7 \%$ (95\% CI: $11.4-18.5 \%)$ were serologically positive for $H$. pylori. Eradication rate by triple therapy with esomeprazole $20 \mathrm{mg}$, amoxicillin $250 \mathrm{mg}$ or $750 \mathrm{mg}$, and clarithromycin $200 \mathrm{mg}$ twice daily for 1 week was $81.1 \%(95 \%$ CI: $64.8-92.0 \%$ ). Of the 25 patients who agreed to this follow-up survey, 4 patients dropped out, due to relocation $(n=1)$, loss to follow-up $(n=1)$ or study withdrawal $(n=2)$. Finally, 21 hemodialysis patients were evaluated for change in serological nutrition markers and plasma ghrelin level at 1 year after $H$. pylori eradication therapy. Hemodialysis patients with failured eradication therapy were received the second-line therapy (esomeprazole, amoxicillin, and metronidazole) for 7 days.

The mean age of hemodialysis patients was $64.4 \pm 9.5$ years, mean body weight (dry weight) was $57.9 \pm 14.5 \mathrm{~kg}$, and mean BMI was $22.6 \pm 4.6 \mathrm{~kg} / \mathrm{m}^{2}$ (Table 1). The causes of end-stage renal disease were chronic glomerulonephritis $(n=10)$, diabetic nephropathy $(n=5)$, nephrosclerosis $(n=2)$, IgA nephropathy $(n=1)$ and others $(n=3)$. The mean duration of hemodialysis was $4.7 \pm 4.5$ years. Endoscopy revealed no peptic ulcer lesions or gastric cancer in any patient. In contrast, all patients had gastric mucosal atrophy with diffuse redness. The severity of gastric mucosal atrophy before eradication therapy was mild $(3 / 21$, $14.3 \%)$, moderate $(5 / 21,23.8 \%)$ and severe $(13 / 21,61.9 \%)$.

Influence of $\boldsymbol{H}$. pylori eradication therapy on plasma ghrelin level. Plasma acyl- and desacyl-ghrelin levels before eradication were $11.6 \pm 8.6 \mathrm{fmol} / \mathrm{ml}$ and $194.9 \pm 117.1 \mathrm{fmol} / \mathrm{ml}$ (Table 1) versus $9.7 \pm 5.1 \mathrm{fmol} / \mathrm{ml}$ and $223.3 \pm 140.8 \mathrm{fmol} / \mathrm{ml}$ after eradication, respectively. No significant differences between plasma acyl- and desacyl-ghrelin levels before and after eradication were observed ( $p=0.274$ and $p=0.455$, respectively) (Table 1$)$.

Influence of $\boldsymbol{H}$. pylori eradication therapy on nutrition markers. Although there were no significant changes in dry weight or BMI before and after eradication, serum cholinesterase level after eradication was significantly higher than that before eradication $(303.2 \pm 76.0 \mathrm{IU} / \mathrm{L}$ vs $287.3 \pm 68.1 \mathrm{IU} / \mathrm{L}, p=0.029)$ (Table 1).

Because plasma ghrelin level is associated with the severity of gastric mucosal atrophy, ${ }^{(23)}$ we evaluated the relationship between nutrition markers and severity of gastric mucosal atrophy to clarify the characteristics of patients whose nutrition markers improved. While nutrition markers (serum total cholesterol, cholinesterase, albumin and GNRI) in patients with severe atrophy did not improve, serum cholesterol levels (before, 196.6 \pm 23.2 $\mathrm{mg} / \mathrm{dl}$; after, $206.1 \pm 25.9 \mathrm{mg} / \mathrm{dl}, p=0.042$ ) and cholinesterase levels (before, $296.9 \pm 70.8 \mathrm{IU} / \mathrm{L}$; after, $316.4 \pm 73.8 \mathrm{IU} / \mathrm{L}, p=$ $0.049)$ were significantly increased after eradication in patients who had mild to moderate atrophy (Table 2).

Serum pepsinogen levels in hemodialysis patients after eradication therapy. Serum PG I and PG II levels and PG I/PG II ratio were $199.5 \pm 102.3 \mathrm{ng} / \mathrm{ml}, 21.5 \pm 9.2 \mathrm{ng} / \mathrm{ml}$ and $9.8 \pm 4.8$, respectively. The serum PG II level was significantly decreased (before, $54.4 \pm 40.3 \mathrm{ng} / \mathrm{ml}$; after, $21.5 \pm 9.2 \mathrm{ng} / \mathrm{ml}, p<0.001$ ) and PG I/II ratio was significantly increased after eradication (before $5.3 \pm 3.1$, after $9.8 \pm 4.8, p<0.001$ ) (Table 1 and Fig. 1). 
Table 1. Characteristics and nutrition markers of hemodialysis patients before and after eradication therapy

\begin{tabular}{lccc}
\hline & Before eradication & After eradication & $p$ value \\
\hline Body weight $(\mathrm{kg})$ & $57.9 \pm 14.5$ & $57.8 \pm 15.2$ & 0.715 \\
Body mass index $\left(\mathrm{kg} / \mathrm{m}^{2}\right)$ & $22.6 \pm 4.6$ & $22.5 \pm 4.8$ & 0.566 \\
Albumin (g/dl) & $4.0 \pm 0.3$ & $4.1 \pm 0.3$ & 0.063 \\
Total cholesterol (mg/dl) & $187.3 \pm 37.2$ & $193.8 \pm 38.5$ & 0.118 \\
Cholinesterase (IU/L) & $287.3 \pm 68.1$ & $303.2 \pm 76.0$ & 0.029 \\
GNRI & $90.6 \pm 7.7$ & $92.1 \pm 7.6$ & 0.055 \\
CRP (mg/dl) & $0.4 \pm 0.8$ & $0.2 \pm 0.2$ & 0.469 \\
Acyl-ghrelin (fmol/ml) & $11.6 \pm 8.6$ & $9.7 \pm 5.1$ & 0.274 \\
Desacyl-ghrelin (fmol/ml) & $194.9 \pm 117.1$ & $223.3 \pm 140.8$ & 0.455 \\
Acyl-ghrelin/total ghrelin & $5.5 \pm 2.5$ & $4.7 \pm 1.8$ & 0.244 \\
Pepsinogen I (ng/ml) & $285.8 \pm 266.0$ & $199.5 \pm 102.3$ & 0.164 \\
Pepsinogen II (ng/ml) & $54.4 \pm 40.3$ & $21.5 \pm 9.2$ & $<0.001$ \\
Pepsinogen I/Il ratio & $5.3 \pm 3.1$ & $9.8 \pm 4.8$ & $<0.001$ \\
\hline
\end{tabular}

Values are presented as mean \pm SD. $P$ value: before vs 1 year after eradication. GNRI, geriatric nutritional risk index; CRP, C-reactive protein.

Table 2. Change in nutrition parameters after $H$. pylori eradication according to severity of gastric mucosal atrophy

\begin{tabular}{|c|c|c|c|c|c|c|}
\hline \multirow{2}{*}{$\begin{array}{l}\text { Severity of gastric mucosal atrophy } \\
\text { Age (years) }\end{array}$} & \multicolumn{3}{|c|}{ Mild/Moderate } & \multicolumn{3}{|c|}{ Severe } \\
\hline & \multicolumn{3}{|c|}{$62.9 \pm 8.8$} & \multicolumn{3}{|c|}{$65.3 \pm 10.1$} \\
\hline Male/Female $(n / n)$ & \multicolumn{3}{|c|}{$5 / 3$} & \multicolumn{3}{|c|}{$6 / 7$} \\
\hline Duration of hemodialysis (years) & \multicolumn{3}{|c|}{$4.6 \pm 5.0$} & \multicolumn{3}{|c|}{$4.7 \pm 4.4$} \\
\hline Body weight (kg) & $61.7 \pm 15.2$ & $61.2 \pm 15.7$ & 0.575 & $55.6 \pm 14.2$ & $55.7 \pm 15.2$ & 0.944 \\
\hline Body mass index $\left(\mathrm{kg} / \mathrm{m}^{2}\right)$ & $23.3 \pm 4.3$ & $23.1 \pm 4.6$ & 0.575 & $22.2 \pm 4.9$ & $22.2 \pm 5.1$ & 0.753 \\
\hline Cholinesterase (IU/L) & $296.9 \pm 70.8$ & $316.4 \pm 73.8$ & 0.049 & $281.4 \pm 68.6$ & $295.2 \pm 79.1$ & 0.182 \\
\hline GNRI & $91.4 \pm 7.9$ & $92.5 \pm 6.5$ & 0.363 & $90.0 \pm 7.8$ & $91.9 \pm 8.4$ & 0.093 \\
\hline CRP (mg/dl) & $0.2 \pm 0.3$ & $0.2 \pm 0.1$ & 0.866 & $0.5 \pm 1.0$ & $0.2 \pm 0.3$ & 0.272 \\
\hline Acyl-ghrelin (fmol/ml) & $9.8 \pm 8.5$ & $9.1 \pm 4.7$ & 0.889 & $12.7 \pm 8.8$ & $10.1 \pm 5.5$ & 0.221 \\
\hline Desacyl-ghrelin (fmol/ml) & $184.0 \pm 131.5$ & $252.9 \pm 201.2$ & 0.575 & $201.6 \pm 112.4$ & $205.1 \pm 92.0$ & 0.65 \\
\hline
\end{tabular}

GNRI, geriatric nutritional risk index; CRP, C-reactive protein.

A

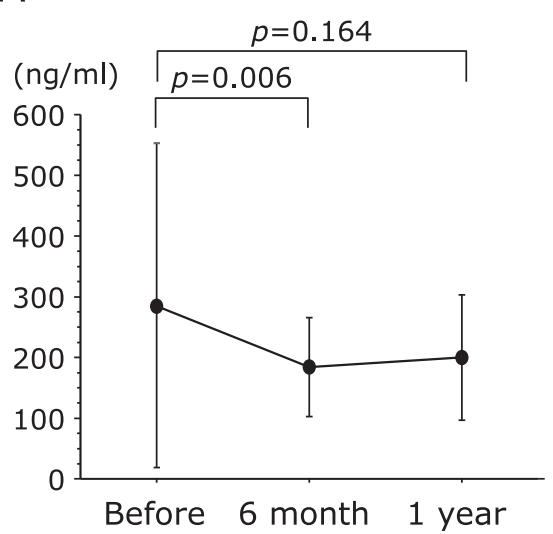

B

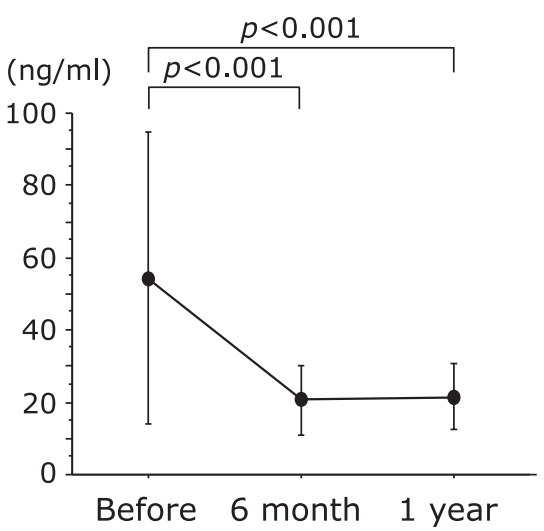

C

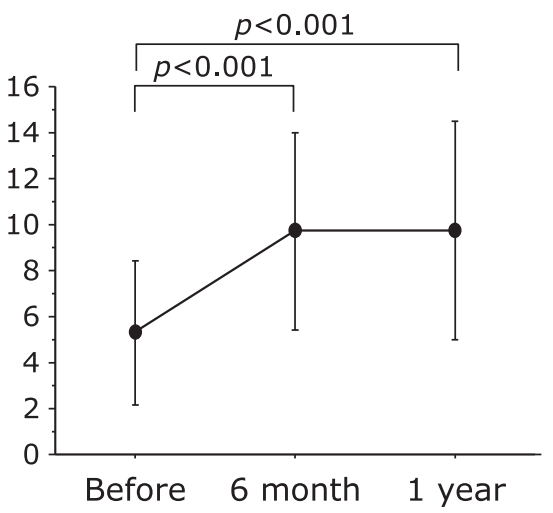

Fig. 1. Serum pepsinogen (PG) I level (A), serum PG II level (B) and PG I/II ratio (C) before and after eradication. Serum PG II levels were significantly decreased and PG I/II ratio was significantly increased after eradication. 


\section{Discussion}

We demonstrated that the serological markers, cholinesterase and cholesterol, increased after $H$. pylori eradication therapy in H. pylori-positive hemodialysis patients, especially in those with gastric mild-moderate atrophy who has ability for recovery of gastric mucosal atrophy after eradication therapy. ${ }^{(28)}$ Therefore, eradication therapy might improve nutritional status in $\mathrm{H}$. pyloripostive hemodialysis patients. Nutritional status is expected to have a markedly greater effect on prognosis in hemodialysis patients than in individuals with nomal renal function. We therefore recommend that hemodialysis patients be checked for $H$. pylori infection and that eradication therapy should be initiated before the progression of gastric atrophy.

Association between $\boldsymbol{H}$. pylori eradication and ghrelin levels in hemodialysis patients. $H$. pylori infection downregulated plasma and gastric mucosal ghrelin levels, and gastric ghrelin mRNA level and number of ghrelin-positive cells significantly increased after eradication. ${ }^{(22,23,29,30)}$ Although it has remained unclear whether ghrelin levels significantly increased after eradication therapy in hemodilysis patients, increasing appetite and food intake was observed in hemodialysis patients with successful eradication. However, we failed to show that plasma ghrelin levels and acyl-ghrelin/total ghrelin ratio after eradication therapy increased compared with before therapy. ${ }^{(31)}$ Because plasma ghrelin levels were reported to have no correlations with expression of gastric ghrelin mRNA or the number of ghrelinpositive cells after eradication therapy, ${ }^{(29)}$ improvement of nutrition markers may cause by increase of gastric ghrelin mRNA and the number of ghrelin-positive cells might increase after eradication therapy. However, we have no sample to measure gastric ghrelin levels and this is limitation of this study. Therefore, we should seek to clarify whether the gastric ghrelin level changes after $H$. pylori eradication therapy in hemodialysis patients.

Association between $\boldsymbol{H}$. pylori eradication and nutrition markers. Nutrition status is regulated by hormonal systems such as ghrelin and leptin. ${ }^{(32)}$ Chronic gastritis affects a variety of endocrine functions of the stomach including the production of the gastrointestinal hormones and neurotransmitters somatostatin, gastrin and ghrelin. ${ }^{(10-12)}$ Patients often develop hyperlipidemia after eradication therapy and their body weight and BMI increase, ${ }^{(19,33-35)}$ suggesting that $H$. pylori is involved in the physiologic regulation of ghrelin and leptin. ${ }^{(36)} \mathrm{PEW}$ is defined as a state of decreased body stores of protein and energy fuels (body protein and fat mass) and is diagnosed if the following three features are present: (1) abnormal nutrition markers (i.e., low serum levels of albumin or cholesterol); (2) reduced body mass (i.e., low or reduced body or fat mass or weight loss with reduced intake of energy); and (3) reduced muscle mass (i.e., muscle wasting or sarcopenia, and reduced mid-arm muscle circumference). ${ }^{(37)}$ In this study, we observed an increase of cholinesterase and cholesterol levels after eradication. Because serum cholesterol level is included in the diagnostic criteria for PEW as a nutrition marker, the increase in cholesterol level after eradication might contribute to improved prognosis in hemodialysis patients. It is possible that the improvement in nutrition status and increase in BMI after eradication therapy might depend not only on an increase of ghrelin but also on another biological mechanisms. Such mechanisms include an increase of gastrointestinal motility, ${ }^{(10,11,38)}$ change in gut microbiome profile, ${ }^{(39)}$ and increase in absorption ability after eradication. ${ }^{(40)}$ Zhang et al. ${ }^{(38)}$ showed that

\section{References}

1 Ikizler TA, Cano NJ, Franch H, et al. Prevention and treatment of protein energy wasting in chronic kidney disease patients: a consensus statement by the International Society of Renal Nutrition and Metabolism. Kidney Int 2013; 84: 1096-1107. in patients with functional dyspepsia, gastric half-emptying times in $H$. pylori-negative patients and eradicated patients were significantly shorter than those in patients who underwent conventional treatment. Betrapally et $a l^{(39)}$ reported that alterations to the intestinal microbiota affect the development of nonalcoholic steatohepatitis by influencing digestion, development of obesity, the immune response, and production of gut hormones. H. pylori eradication therapy changed the gastrointestinal microbiota. ${ }^{(41)}$ Therefore, eradication therapy might have improved nutrition status. A study to examine whether the long-term prognosis of hemodialysis patients is improved by eradication therapy will be required to investigate this hypothesis further.

\section{Conclusion}

In this small preliminary study, H. pylori eradication did increase levels of nutritional markers in patients with mild and moderate gastric atrophy. H. pylori eradication therapy might therefore contribute not only to the prevention of gastrointestinal disease, but also to improvements in the prognosis of hemodialysis patients. We recommend that the $H$. pylori status of hemodialysis patients be checked. We plan to conduct a long-term follow-up survey to investigate whether the prognosis of $H$. pylori-positive hemodialysis patients improves after eradication; and if so, whether this improvement is related to plasma ghrelin level and nutritional markers.

\section{Author Contributions}

Ichikawa H, Sugimoto M, Sakao Y, Sahara S, Ohashi N and Yasuda $\mathrm{H}$ contributed to study conception and design; Ichikawa H, Sakao Y, Sahara S, Ohashi N, Sano K, Tadokoro S, Azekura H, Shimomura A, Yamashita F, Sugiyama D and Fukuta K contributed to data acquisition; Ichikawa H, Sugimoto M, Sakao Y and Ohashi $\mathrm{N}$ contributed to data analysis and interpretation; Ichikawa $\mathrm{H}$ and Sugimoto $\mathrm{M}$ wrote the paper; Kato A, Sugimoto $\mathrm{K}$ and Furuta $\mathrm{T}$ edited the paper.

\section{Acknowledgments}

This study was supported by a Grant-in-Aid from the Ministry of Education, Culture, Sports, Science and Technology of Japan (15K08948) and a Grant-in-Aid from the Japanese Association of Dialysis Physicians (JADP Grant 2014-2).

The authors would like to thank the nephrologists (Sato D, Tsuji $\mathrm{N}$, Ishigaki S, Naito Y, Iwakura T, Isobe S, Ono M, Fujikura T, Tsuji $\mathrm{T}$ and Fujigaki $\mathrm{Y}$ ) at Hamamatsu University School of Medicine for their assistance with sample collection.

\section{Abbreviations \\ BMI body mass index \\ H. pylori Helicobacter pylori \\ PEW protein-energy wasting \\ PG pepsinogen}

\section{Conflict of Interest}

No potential conflicts of interest were disclosed.

2 Kovesdy CP, George SM, Anderson JE, Kalantar-Zadeh K. Outcome predictability of biomarkers of protein-energy wasting and inflammation in moderate and advanced chronic kidney disease. Am J Clin Nutr 2009; 90: $407-414$. 
3 Carrero JJ, Nakashima A, Qureshi AR, et al. Protein-energy wasting modifies the association of ghrelin with inflammation, leptin, and mortality in hemodialysis patients. Kidney Int 2011; 79: 749-756.

4 Mak RH, Cheung WW. Is ghrelin a biomarker for mortality in end-stage renal disease? Kidney Int 2011; 79: 697-699.

5 Kojima M, Hosoda H, Date Y, Nakazato M, Matsuo H, Kangawa K. Ghrelin is a growth-hormone-releasing acylated peptide from stomach. Nature 1999; 402: 656-660.

6 Pradhan G, Samson SL, Sun Y. Ghrelin: much more than a hunger hormone. Curr Opin Clin Nutr Metab Care 2013; 16: 619-624.

7 Akamizu T, Kangawa K. Ghrelin for cachexia. J Cachexia Sarcopenia Muscle 2010; 1: 169-176.

8 Suzuki H, Matsuzaki J, Hibi T. Ghrelin and oxidative stress in gastrointestinal tract. J Clin Biochem Nutr 2011; 48: 122-125.

9 Arakawa M, Suzuki H, Minegishi Y, et al. Enhanced ghrelin expression and subsequent acid secretion in mice with genetic $\mathrm{H}_{2}$-receptor knockout. $J$ Gastroenterol 2007; 42: 711-718.

10 Suzuki H, Matsuzaki J, Fukushima Y, et al. Randomized clinical trial: rikkunshito in the treatment of functional dyspepsia--a multicenter, doubleblind, randomized, placebo-controlled study. Neurogastroenterol Motil 2014; 26: 950-961.

11 Suzuki H, Moayyedi P. Helicobacter pylori infection in functional dyspepsia. Nat Rev Gastroenterol Hepatol 2013; 10: 168-174.

12 Iwasaki E, Suzuki H, Mogami S, Hibi T. Enhanced gastric ghrelin-induced c-Kit protein expression in rats with gastric outlet obstruction. Dig Dis Sci 2012; 57: 3308-3309.

13 Mogami S, Suzuki H, Fukuhara S, Matsuzaki J, Kangawa K, Hibi T. Reduced ghrelin production induced anorexia after rat gastric ischemia and reperfusion. Am J Physiol Gastrointest Liver Physiol 2012; 302: G359-G364.

14 Chou CC, Bai CH, Tsai SC, Wu MS. Low serum acylated ghrelin levels are associated with the development of cardiovascular disease in hemodialysis patients. Intern Med 2010; 49: 2057-2064.

15 Wynne K, Giannitsopoulou K, Small CJ, et al. Subcutaneous ghrelin enhances acute food intake in malnourished patients who receive maintenance peritoneal dialysis: a randomized, placebo-controlled trial. J Am Soc Nephrol 2005; 16: 2111-2118.

16 Ashby DR, Ford HE, Wynne KJ, et al. Sustained appetite improvement in malnourished dialysis patients by daily ghrelin treatment. Kidney Int 2009; 76: 199-206.

17 Sugimoto M, Sakai K, Kita M, Imanishi J, Yamaoka Y. Prevalence of Helicobacter pylori infection in long-term hemodialysis patients. Kidney Int 2009; 75: 96-103.

18 Sugimoto M, Yamaoka Y. Review of Helicobacter pylori infection and chronic renal failure. Ther Apher Dial 2011; 15: 1-9.

19 Furuta T, Shirai N, Xiao F, Takashima M, Hanai H. Effect of Helicobacter pylori infection and its eradication on nutrition. Aliment Pharmacol Ther 2002; 16: 799-806.

20 Jang EJ, Park SW, Park JS, et al. The influence of the eradication of Helicobacter pylori on gastric ghrelin, appetite, and body mass index in patients with peptic ulcer disease. J Gastroenterol Hepatol 2008; 23 Suppl 2: S278S285.

21 Kawashima J, Ohno S, Sakurada T, et al. Circulating acylated ghrelin level decreases in accordance with the extent of atrophic gastritis. J Gastroenterol 2009; 44: 1046-1054.

22 Suzuki H, Masaoka T, Hosoda H, et al. Helicobacter pylori infection modifies gastric and plasma ghrelin dynamics in Mongolian gerbils. Gut 2004; 53: $187-194$.
23 Ichikawa H, Sugimoto M, Sakao Y, et al. Relationship between ghrelin, Helicobacter pylori and gastric mucosal atrophy in hemodialysis patients. World J Gastroenterol 2016; 22: 10440-10449.

24 Yamada K, Furuya R, Takita T, et al. Simplified nutritional screening tools for patients on maintenance hemodialysis. Am J Clin Nutr 2008; 87: 106-113.

25 Bouillanne O, Morineau G, Dupont C, et al. Geriatric Nutritional Risk Index: a new index for evaluating at-risk elderly medical patients. Am J Clin Nutr 2005; 82: 777-783.

26 Kimura K, Takemoto T. An endoscopic recognition of the atrophic border and its significance in chronic gastritis. Endoscopy 1969; 1: 87-97.

27 Miki K, Ichinose M, Ishikawa KB, et al. Clinical application of serum pepsinogen I and II levels for mass screening to detect gastric cancer. Jpn J Cancer Res 1993; 84: 1086-1090.

28 Kodama M, Murakami K, Okimoto T, et al. Ten-year prospective follow-up of histological changes at five points on the gastric mucosa as recommended by the updated Sydney system after Helicobacter pylori eradication. $J$ Gastroenterol 2012; 47: 394-403.

29 Osawa H, Kita H, Ohnishi H, et al. Changes in plasma ghrelin levels, gastric ghrelin production, and body weight after Helicobacter pylori cure. J Gastroenterol 2006; 41: 954-961.

30 Tatsuguchi A, Miyake K, Gudis K, et al. Effect of Helicobacter pylori infection on ghrelin expression in human gastric mucosa. Am J Gastroenterol 2004; 99: 2121-2127.

31 Masaoka T, Suzuki H, Imaeda H, et al. Long-term strict monitoring of plasma ghrelin and other serological markers of gastric diseases after Helicobacter pylori eradication. Hepatogastroenterology 2005; 52: 1-4.

32 Elmquist JK, Maratos-Flier E, Saper CB, Flier JS. Unraveling the central nervous system pathways underlying responses to leptin. Nat Neurosci 1998; 1: $445-450$.

33 Kamada T, Hata J, Kusunoki H, et al. Eradication of Helicobacter pylori increases the incidence of hyperlipidaemia and obesity in peptic ulcer patients. Dig Liver Dis 2005; 37: 39-43.

34 Lane JA, Murray LJ, Harvey IM, Donovan JL, Nair P, Harvey RF. Randomised clinical trial: Helicobacter pylori eradication is associated with a significantly increased body mass index in a placebo-controlled study. Aliment Pharmacol Ther 2011; 33: 922-929.

35 Azuma T, Suto H, Ito Y, et al. Eradication of Helicobacter pylori infection induces an increase in body mass index. Aliment Pharmacol Ther 2002; 16 Suppl 2: 240-244.

36 Francois F, Roper J, Joseph N, et al. The effect of $H$. pylori eradication on meal-associated changes in plasma ghrelin and leptin. BMC Gastroenterol 2011; 11: 37

37 Fouque D, Kalantar-Zadeh K, Kopple J, et al. A proposed nomenclature and diagnostic criteria for protein-energy wasting in acute and chronic kidney disease. Kidney Int 2008; 73: 391-398.

38 Zhang CL, Geng CH, Yang ZW, et al. Changes in patients' symptoms and gastric emptying after Helicobacter pylori treatment. World J Gastroenterol 2016; 22 : 4585-4593.

39 Betrapally NS, Gillevet PM, Bajaj JS. Changes in the intestinal microbiome and alcoholic and nonalcoholic liver diseases: causes or effects? Gastroenterology 2016; 150: 1745-1755.e3.

40 Serin E, Gümürdülü Y, Ozer B, Kayaselçuk F, Yilmaz U, Koçak R. Impact of Helicobacter pylori on the development of vitamin B12 deficiency in the absence of gastric atrophy. Helicobacter 2002; 7: 337-341.

41 Li L, Zhou X, Xiao S, Ye F, Zhang G. The effect of Helicobacter pylori eradication on the gastrointestinal microbiota in patients with duodenal ulcer. J Gastrointestin Liver Dis 2016; 25: 139-146. 